\title{
Fatigue behaviour of composite adhesive lap joints
}

\author{
J.A.M Ferreira ${ }^{\mathrm{a}, *}$, P.N Reis ${ }^{\mathrm{b}}$, J.D.M Costa ${ }^{\mathrm{a}}$, M.O.W Richardson ${ }^{\mathrm{c}}$ \\ ${ }^{a}$ Department of Mechanical Engineering, University of Coimbra, Pinhal de Marraces 3030 Coimbra, Portugal \\ ${ }^{\mathrm{b}}$ Department of Electromechanical Engineering, UBI, Covilhã, Portugal \\ ${ }^{\mathrm{c}}$ Department of Mechanical and Manufacturing Engineering, Portsmouth University, UK
}

Received 2 November 2000; received in revised form 17 March 2002; accepted 30 April 2002

\begin{abstract}
This paper is concerned with a fatigue study of composite adhesive lap joints produced from a bi-directional woven E-glass fibres and polypropylene. The adhesive used was a Bostik 7452 (Rubber \& Plastics Grade) ethyl cyanoacrylate type. The effects of layer orientation, lap joint length and water immersion on the fatigue behaviour were studied. The specimens were immersed in water during periods until ninety days for controlled temperatures of 20,40 and $70{ }^{\circ} \mathrm{C}$. The results are presented in the form of curves of stress amplitude versus number of cycles to failure and also in the form of number of cycles to failure against time to exposure in water for fixed stress amplitudes. The fatigue damage and failure mechanisms were analysed and discussed. The joint shows that creep deformation within the temperature range of this study was probably the mainly cause of the dynamic stiffness reduction observed. (C) 2002 Elsevier Science Ltd. All rights reserved.
\end{abstract}

Keywords: A. Adhesive joints; B. Fatigue; B. Creep; Environment effect

\section{Introduction}

Bonded joints promote a more uniform stress distribution than other methods of joining, which rely on single joint contacts, such as bolting, riveting and spot welding, where high stress concentrations occur. Bonding gives also a smoother appearance to the final assembly and the ability to join different materials in complex shapes.

The response of bonded joints to fatigue loading has been extensively researched in the last years concluding that the failure generally initiates at the adhesive [1-3] for moderate stress levels. The fatigue life is strongly influenced by the profile of the edges of the joint. The initiation of small cracks at the edges points represent the major part of the fatigue life [4].

The static and fatigue properties of polymeric adhesives can be influenced by environmental conditions such as: temperature, moisture and chemical agents. It has been shown that thermal effects, whether due to

\footnotetext{
* Corresponding author. Tel.: + 351-2397-90700; fax: +351-23979701.

E-mail address: martins.ferreira@mail.dem.uc.pt (J.A.M Ferreira).
}

mismatch of the adherends or to adhesive contraction by temperature of cure, lead to significant changes in the stress state of lap joints [5]. Harris and Fay [4] also concluded that the temperature $T_{\mathrm{g}}$ of the adhesive should be above the maximum temperature expected in service.

It has been also verified that the strength of joints usually decreases in presence of humidity and time of exposure [6-8]. Also the debonding of adhesives caused by cleavage of cracks increases with the water presence in form of liquid or vapour [9]. This is the result of the hydrophilic nature of adhesives, which is caused by the polar groups, needed to confer adhesive properties on polymeric materials. Therefore chemical degradation of the adhesive, substrate and chemical bonds across the interface is possible as a result of interaction with water. The water can enter in the adhesive and then attack it by diffusion through the adhesive and adherent, and finally transport along the interface and move by capillary action through cracks in the adhesive. The moisture can not only affect the adhesive but also the mechanical behaviour of the adherend composite. However no significant effect of moisture was observed on the static strength [10], and fatigue crack growth [11] of laminate composites. 
For this study a single lap shear joint was chosen. This type of joint promotes low fatigue performance because of the high shear and peel stress concentrations that arise in the adhesive layer at the ends of the overlap [12]. The objectives of the study were to test the static and fatigue performance of the adhesive used with polypropylene based composites and the ageing effect caused by the presence of water. It was considered important to associate the simultaneous effect of temperature and water. Also the creep deformation was studied because it can be related with fatigue threshold behaviour [4].

\section{Materials and experimental procedures}

Composite sheets were obtained from multi-layers of Vertotex "Twintex T PP", which were processed at $190{ }^{\circ} \mathrm{C}$ in a mould under pressure of 5 bar, for $10 \mathrm{~min}$. Each sheet was made up of seven woven balanced bidirectional ply layers. The overall dimensions of the plates were $160 \times 250 \times 3 \mathrm{~mm}$ with a fibre volume fraction of 0.338 , as expected and reported by the manufacturer.

Two types of plate were manufactured for this study. In one of them all the layers had one of the fibre orientations coinciding with the load direction, $0^{\circ} / 0^{\circ} / 0^{\circ} / 0^{\circ} / 0^{\circ} / 0^{\circ} / 0^{\circ}$. The other had the following ply orientation: $+45^{\circ} /-45^{\circ} /$ $+45^{\circ} / 0^{\circ} /+45^{\circ} /-45^{\circ} /+45^{\circ}$. These two types of laminates will be referred to as $0 / 0$ and $+45 /-45 / 0$, respectively. The specimens used were prepared from these thin plates. The geometry and dimensions of the tension specimens are shown in Fig. 1. The parameter $\ell$ is the length of bond. The fatigue sample geometry was similar, employing a "dog bone" type specimen design.

The adhesive used was Bostik 7452-Super Glue 4, Rubber \& Plastics Grade ethyl cyanoacrylate type, while the primer was Bostik 7480-Super Glue 4 based on n-heptane. To obtain good adhesion it was necessary, first to clean by an ultra-sonic process and then put the specimens for one hour in a bath of trichlorethylene. The bond thickness was kept similar for all the specimens. The samples were visually checked before testing. Later the thickness was more accurately measured using "surface roughness" equipment. An average value of $0.1 \mathrm{~mm}$ was obtained without significant dispersion. In some cases this parameter does not have a significant influence on the fatigue behaviour of adhesive joints [13].

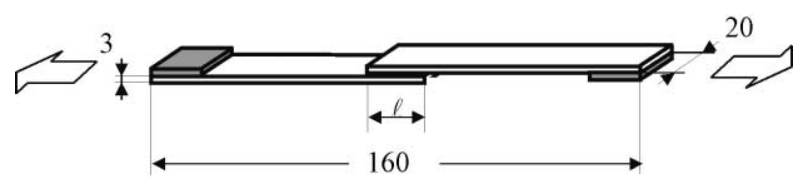

Fig. 1. Geometry and dimensions of the specimen (dimensions in $\mathrm{mm}$ ).
The tensile properties were obtained using an electromechanical Instron Universal Testing machine. The fatigue tests were carried out on a servo-hydraulic Instron machine in constant amplitude load. All fatigue tests were performed in tension with stress ratio of 0.025 and a frequency of $10 \mathrm{~Hz}$, at room temperature. During some of the fatigue tests the temperature rise was measured using two thermocouples placed near the bond edges. The average temperature rise was $4-6{ }^{\circ} \mathrm{C}$ during stable fatigue phase reaching about $10{ }^{\circ} \mathrm{C}$ only at final failure, which means that these values are able to promote only negligible decrease in fatigue life. The creep machine was a weight system type and was equipped with a temperature chamber, a displacement gauge and data acquisition equipment.

\section{Results and discussion}

Four series of fatigue tests were carried out at room temperature to study the influence of the layer distribution and the joint length. The results are plotted in Fig. 2
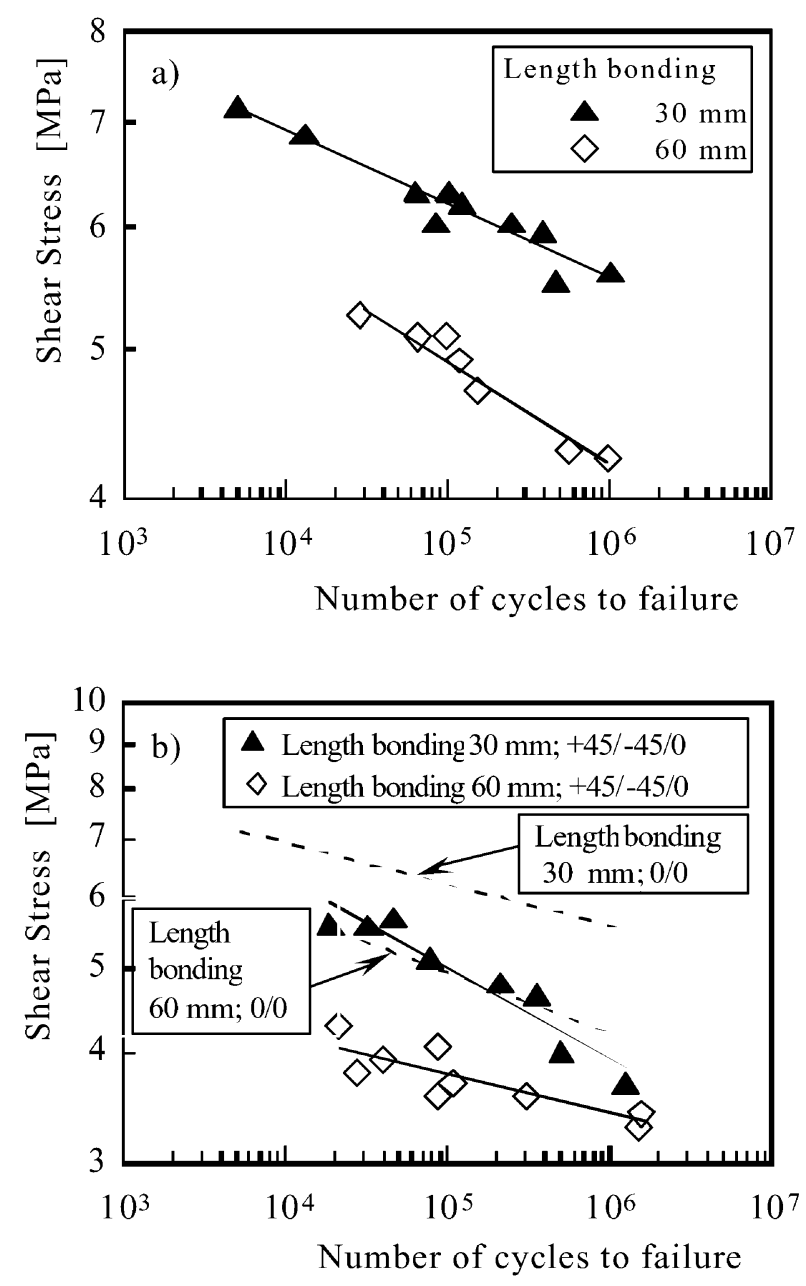

Fig. 2. Influence of the bonding length on the fatigue strength; (a) $0 / 0$ laminate adherent, (b) $+45 /-45 / 0$ laminate adherent. 
in terms of nominal shear stress versus the number of cycles to failure. Fig. $2 \mathrm{a}$ and $\mathrm{b}$ shows the results concerned with $0 / 0$ and $+45 /-45 / 0$ laminate adherent, respectively. In Fig. $2 b$ the mean curves obtained for the 0/0 laminate were also superposed for comparison. For both ply orientations the results show that the fatigue strength was higher for a joint length of $30 \mathrm{~mm}$ than for the joints with $60 \mathrm{~mm}$, being in terms of medium values about $30 \%$. The effect of the lap length on the static mechanical properties was also observed and was due to changes of peel stress values. Changes in fatigue strength caused by bond length followed the same tendency observed with static strength according to the results obtained and indicated in Table 1.The joints with $0 / 0$ adherent exhibited higher fatigue strength than the $+45 /-45 / 0$ laminates, being in terms of medium values of about $30 \%$. In fact the stiffness of the joints will increase with the proportion of fibres aligned in the load direction and then the deformation of the adherent will decrease. This trend agrees with the study of Mathews and Tester [14]. They concluded that strength increases with the proportion of $0^{\circ}$ fibres, leading to the greatest strength in specimens with all plies at $0^{\circ}$.

The influence of water exposure on the static and fatigue strength of the joint was analysed. For this purpose a series of specimens, manufactured with $+45 /$ $-45 / 0$ laminate adherent and $30 \mathrm{~mm}$ bond length, were immersed in a tank full with water which kept at constant temperature. Two parameters were considered, the water temperature, which was rigorously controlled with an error less than $1{ }^{\circ} \mathrm{C}$, and the time of immersion. Three water temperatures were considered: 20; 40 and $70{ }^{\circ} \mathrm{C}$; carrying out three static tests for each condition. In Fig. 3 are plotted the results obtained for the shear strength versus number of days of immersion in water at 20 and $40{ }^{\circ} \mathrm{C}$. Extended immersion causes an important decrease in static strength at both water temperatures. This loss of the strength is about $30 \%$ and occurs at both temperatures. However, the behaviour for immersion times lesser than 45 days depends on the water temperature. The different must be because of the equilibrium points of water uptake for the two temperatures. At $40{ }^{\circ} \mathrm{C}$ the degradation caused by water attack is quicker than for a temperature of $20^{\circ} \mathrm{C}$. In the first case a sudden loss of static strength was observed after a short immersion time of about 15 days, but after this no influence on the strength was observed. At $20{ }^{\circ} \mathrm{C}$ no

Table 1

Average values of static strength

\begin{tabular}{lll}
\hline Laminate adherent layer & Bond length $(\mathrm{mm})$ & Shear strength (MPa) \\
\hline $0 / 0$ & 30 & 12.3 \\
$0 / 0$ & 60 & 10.6 \\
$+45 /-45 / 0$ & 30 & 13.4 \\
$+45 /-45 / 0$ & 60 & 7.5 \\
\hline
\end{tabular}

influence was observed on static strength for 15 days. However, after this period the shear stress decreased about 30\% until 45 days, thereafter the static strength remained nearly constant. The final equilibrium reached by the shear strength is probably related with resin degradation caused by moisture absorption since the water can enter in the composite not only by diffusion but also by capillary action along the fibre-matrix interface [15].

Fig. 4 compares the fatigue strength of degraded adhesive joints having subjected them to 30 days immersion in water at $40{ }^{\circ} \mathrm{C}$ and 8 days in water at $70{ }^{\circ} \mathrm{C}$. For the case of water immersion at $40{ }^{\circ} \mathrm{C}$ only a reduced influence in fatigue strength was observed. The damaging effect of water at $40{ }^{\circ} \mathrm{C}$ showed a slight trend to increase with the stress level. Many adhesive joints are affected by water that can enter in to the joint interface by diffusion and affect the physical and mechanical properties [16,17]. In other words some adhesives exhibits good fatigue performance in a laboratory high humidity environment [18]. This was also observed with the adhesive used in the present

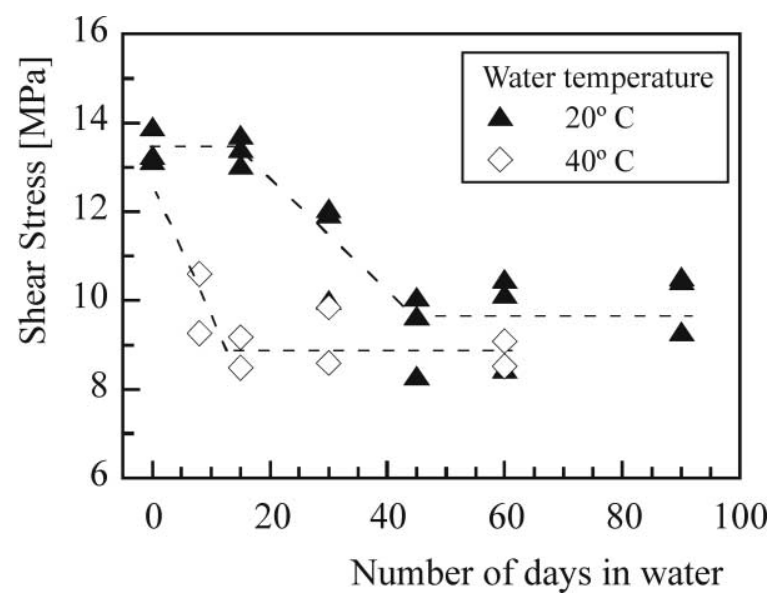

Fig. 3. Shear strength versus number of days in water.

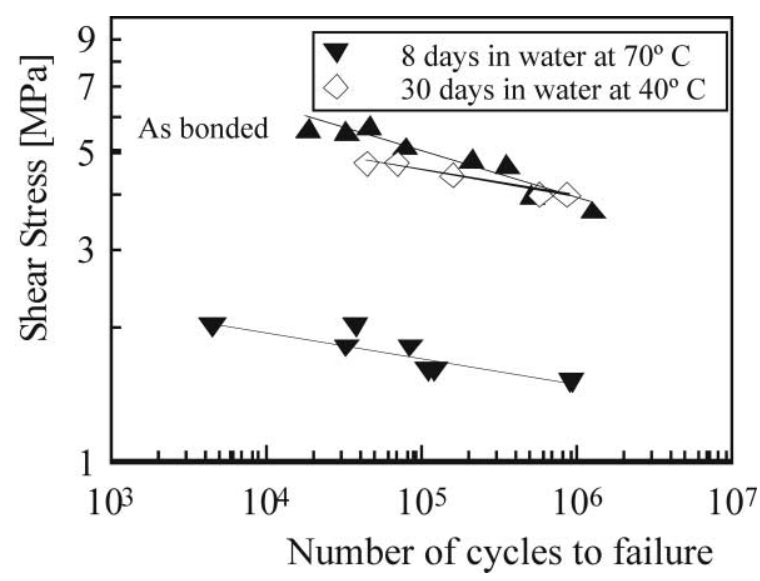

Fig. 4. Influence of water damage on the S-N curves. 
work. Fig. 4 shows not only the effect of the water but also its temperature. As expected the increase in temperature promoted greater diffusion of water and affected the fatigue strength of the polymeric adhesive [5]. In the present investigation only a slight effect of temperature was observed at $40{ }^{\circ} \mathrm{C}$, but an enormous loss of fatigue strength was observed at $70{ }^{\circ} \mathrm{C}$. This temperature was above the critical upper temperature limit of the present adhesive.

Fig. 5 shows the influence of the number of days of water immersion on the number of cycles to failure at a fixed shear stress of $4.7 \mathrm{MPa}$. Two water temperatures were analysed: 20 and $40{ }^{\circ} \mathrm{C}$. A comparison of Figs. 4 and 5 shows that the effect of water observed on the fatigue life for a fixed stress is much more pronounced than on the fatigue strength. In spite of the observed scatter, the fatigue life at a stress of $4.7 \mathrm{MPa}$ is at least four times lower for the case of water immersion at a temperature of $40^{\circ} \mathrm{C}$. Fig. 5 shows some variations due to scatter in the results, but no significant influence of the exposition time on the fatigue life was observed.

The fatigue failure mechanisms were examined by optical microscopy, surface roughness analysis and scanning electronic microscopy (Philips XL30). The objective was to relate the failure mechanisms with fatigue strength. Surface roughness was analysed using a topography measuring station (Mahr) equipped with a laser sensor (Rodenstock RM 600-S). Two limiting mechanisms can be defined: I-the separation of the polymer (PP) associated with the adherent composite and the adhesive caused by water diffusion or deficient bond adherence, II-separation between the fibre and the polymer within the composite. Fig. 6 shows a typical surface photo morphology and roughness profile for the two failure types. In many situations a mixed failure mode was observed. Types I and II were related to the lower and higher fatigue strength tests, respectively. Type I (Fig. 6a) was observed in joints damaged by

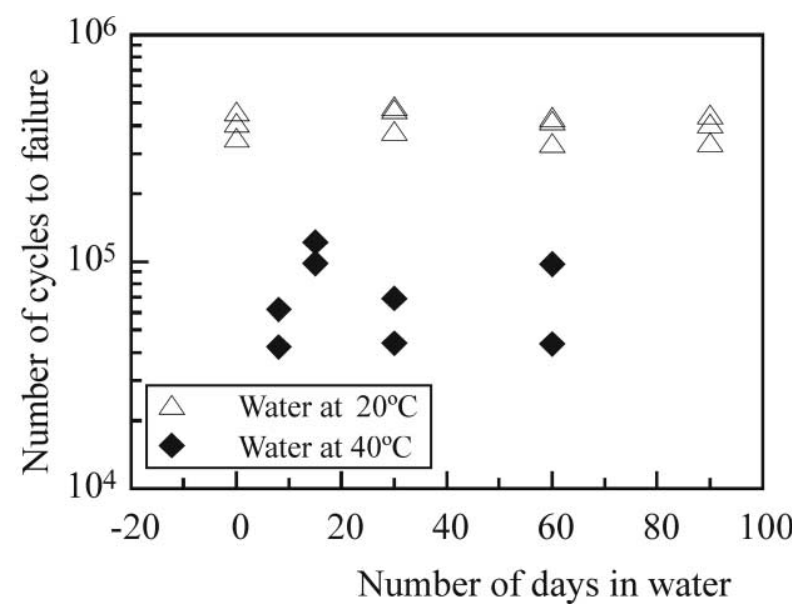

Fig. 5. Influence of water immersion time on the fatigue life for a fixed stress range of $4.7 \mathrm{MPa}$. immersion in water at $70{ }^{\circ} \mathrm{C}$ and in other situations without perfect adherence between the bond and adherent. The failure surface shows a low roughness profile and "depth steps" equivalent to bond thickness. Type II (Fig. 6b) was observed in situations where there was perfect adherence between the adhesive and adherent even after water immersion. In this case the failure occurs at the fibre/adherent polymer interface by action of peel and shear stresses. The failure surface shows a higher roughness profile and deeps steps than in failure Type I.

Periodically, during fatigue tests, the load and correspondent displacement of the specimens, were monitored during some cycles. Two hundred of points per cycle were recorded from which the shear stress versus bond strain was computed and plotted for each cycle. These plots were nearly linear functions. The shear stiffness modulus, $G$, defined by the ratio between the shear stress in the bond and the axial strain, was then derived from the stress-strain curves by linear regression. The stiffness modulus can be used as a fatigue damage parameter of the joint. Fig. 7 plots $G / G_{0}$ versus $N / N_{\mathrm{f}}$, where $G_{0}$ is the initial value of $G, N$ the current number of cycles and $N_{\mathrm{f}}$ is the number of cycles to failure, for joints "as bonded" and also joints tested after immersion in water at $40{ }^{\circ} \mathrm{C}$ and $70{ }^{\circ} \mathrm{C}$.

The results shown in this figure present a trend similar with the typical behaviour observed for the stiffness modulus in the base material $[19,20]$ and other bond joints [4]. A drop in stiffness modulus of about $5 \%$ was observed during the first $10 \%$ of fatigue cycles, followed by a slow decrease until $90-95 \%$ of fatigue life. Thereafter occurs other pronounced decrease of the stiffness modulus until the final failure. Despite some scatter in results, similar trends were observed for the three test conditions. The reduction in dynamic stiffness observed during the first two stages does not show significant differences between the three test series plotted in Fig. 7 and occurs with no evidence of crack growth. This behaviour can be attributed to the creep of the joint and may be thought of as a crack initiation phase [4]. Thus, it was decided to study the creep behaviour of the joint using $0 / 0$ laminate adherent joints, with $l=25 \mathrm{~mm}$, within the temperature ranged from 20 to $50{ }^{\circ} \mathrm{C}$.

Fig. 8 plots the creep strain defined by the ratio between the longitudinal elongation of the gauge and the base length of the gauge (which corresponds with bond length; $l=25 \mathrm{~mm}$ ) against the time for a limited number of the tests. The results of tests for 5.5 and 5.8 $\mathrm{MPa}$ and at different temperatures are plotted. The creep deformation presents three stages. The intermediate stage is nearly linear and is also the most predominant. The shape of the creep curves are the inverse of the loss of dynamic stiffness plotted curves in Fig. 6 . The results show that strain rate and failure time are strongly dependent on temperature and stress. By 
comparison of Figs. 8 and 2a it can be concluded that for the stress ranges used for fatigue testing the joints were highly susceptible to creep, even at room temperature. Thus the reduction in dynamic stiffness observed in the first two stages in Fig. 7 can be attributed to creep deformation.

Creep results for the tests carried out until rupture are indicated in Table 2. These results were analysed using the classic time-temperature parameters [21] and a best

(a)
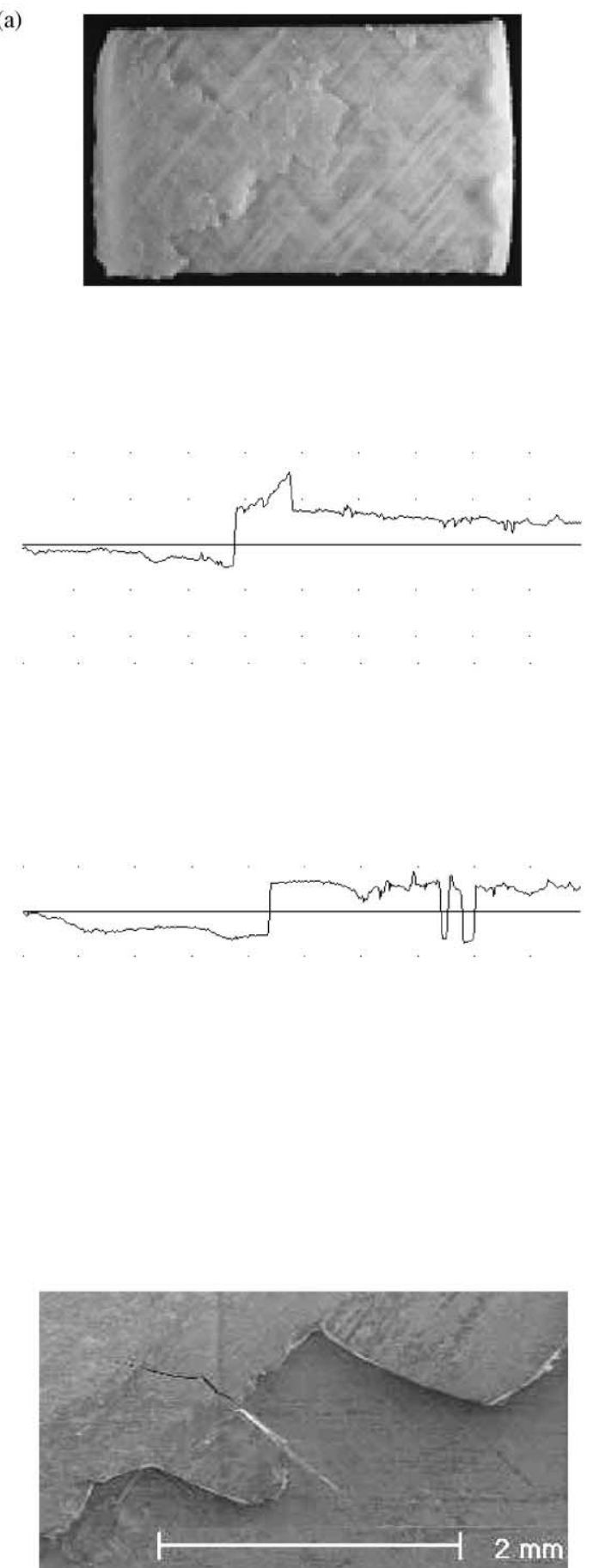

fit was obtained using the Manson-Haferd parameter, defined as:

$P_{\mathrm{MH}}=\frac{T-T_{a}}{\log t_{\mathrm{r}}-\log t_{a}}$

Where: $P_{\mathrm{MH}}$ is the Manson-Haferd parameter, $T$ the temperature in Kelvins, $t_{\mathrm{r}}$ the rupture time in hours, $T_{a}$ and $t_{a}$ material constants.

(b)
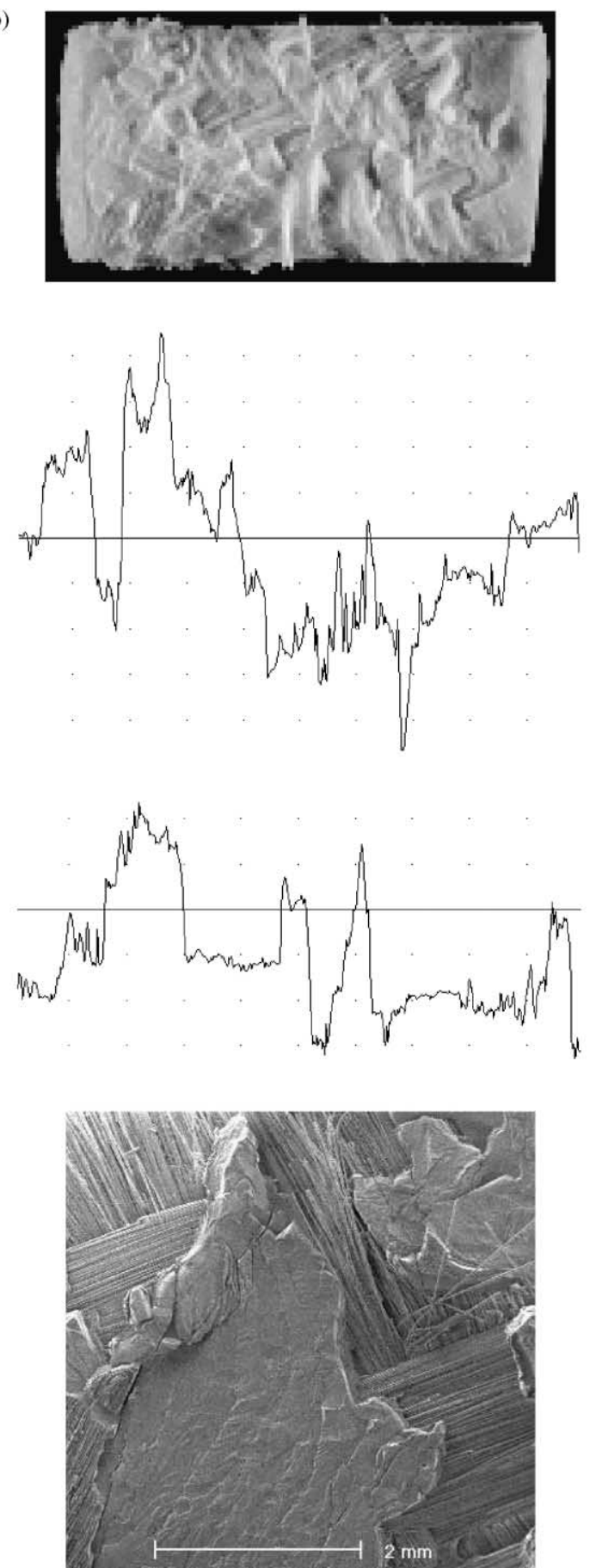

Fig. 6. Fatigue failure details; (a) failure type I, (b) failure type II. 


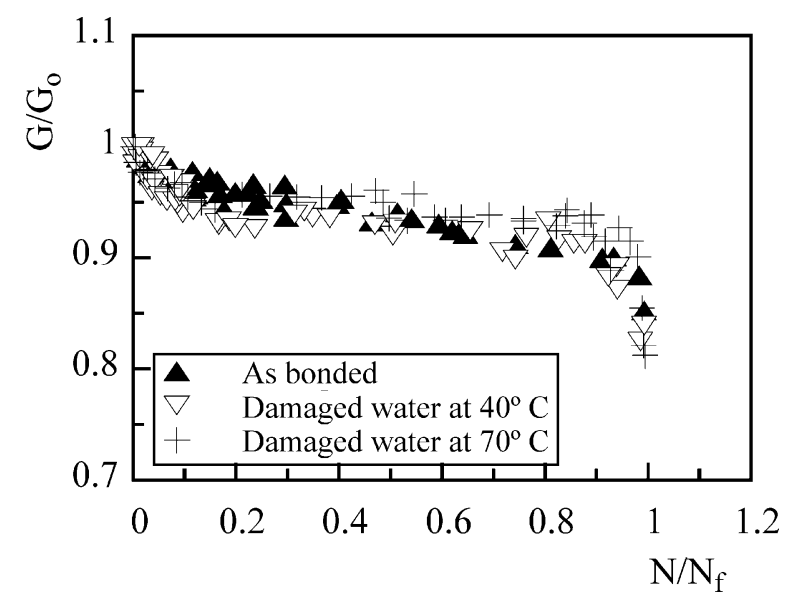

Fig. 7. $G / G_{0}$ against the normalised number of cycles $N / N_{\mathrm{f}} .+45 /-45 /$ 0 laminate adherent joints.

Table 2

Creep results

\begin{tabular}{lll}
\hline $\begin{array}{l}\text { Stress } \\
(\mathrm{MPa})\end{array}$ & $\begin{array}{l}\text { Temperature } \\
(\mathrm{K})\end{array}$ & $\begin{array}{l}\text { Time to } \\
\text { rupture (h) }\end{array}$ \\
\hline 6 & 294 & 10.97 \\
5.5 & 294 & 345.8 \\
5.5 & 294 & 360.1 \\
5.8 & 298 & 122.8 \\
5.8 & 298 & 95.1 \\
5.5 & 303 & 98.5 \\
5.5 & 303 & 89.7 \\
5.8 & 303 & 3.6 \\
5.8 & 303 & 4.5 \\
5.7 & 313 & 9.7 \\
5.5 & 313 & 7.2 \\
5.8 & 313 & 0.35 \\
5.0 & 323 & 82.5 \\
5.0 & 323 & 89.1 \\
\hline
\end{tabular}

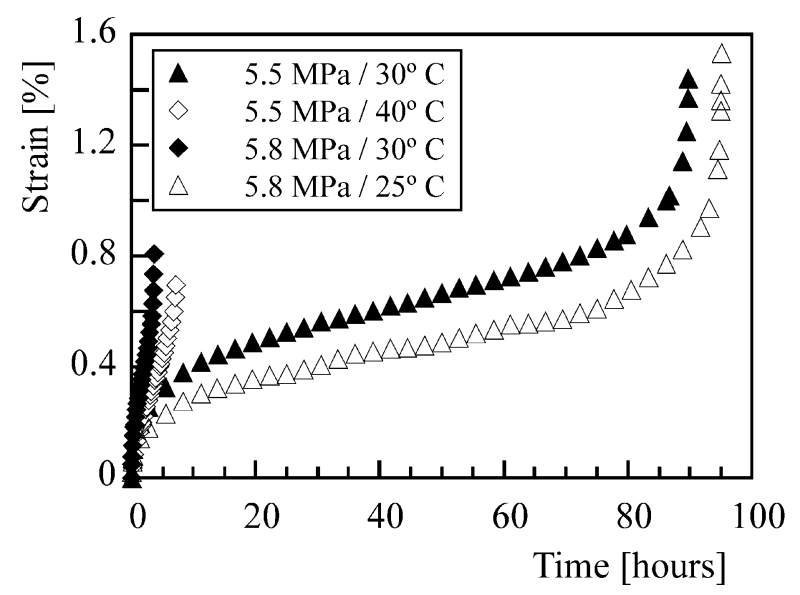

Fig. 8. Typical strain versus time creep curves.

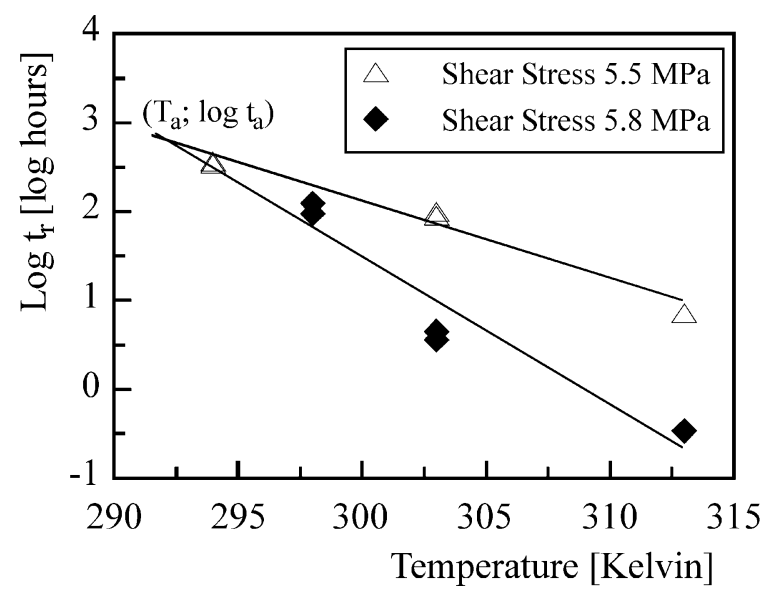

Fig. 9. Plot for determination of the constant of $P_{\mathrm{MH}}$ parameter.

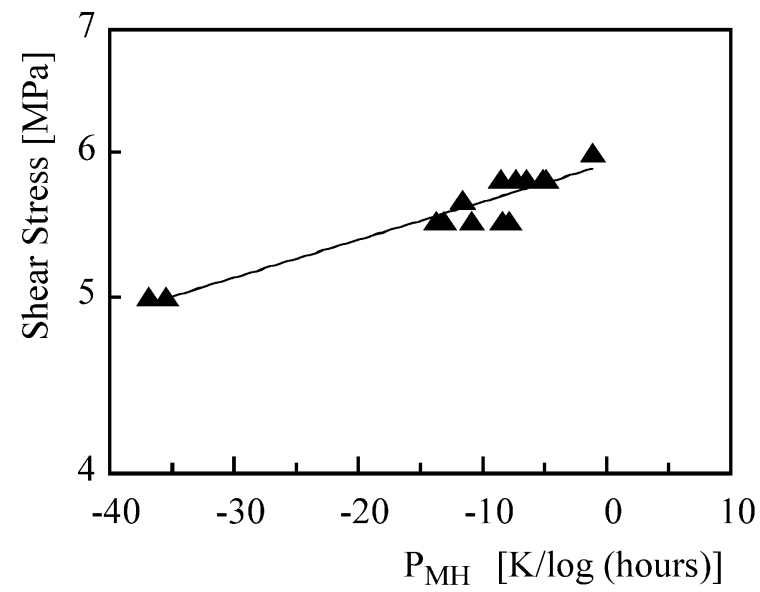

Fig. 10. Shear stress against $P_{\mathrm{MH}}$ parameter.

$\left(T_{a}, \log t_{a}\right)$ can be assumed as the convergence point of a family of straight lines for constant stress in the plot of $T$ versus $\log t_{r}$. This plot is presented in Fig. 9 for the stresses of 5.5 and $5.8 \mathrm{MPa}$. The convergence point is: $T_{a}=292 \mathrm{~K} ; \log t_{a}=2.79$. In Fig. 10 are plotted the $\log$ (stress) against $P_{\mathrm{MH}}$ obtained from the creep tests. In spite of some significant scatter, the results are reasonably fitted (with a correlation coefficient of 0.96 ) by the equation,

$\sigma=5.9266 \mathrm{e}^{0.0049 P_{\mathrm{MH}}}$

\section{Conclusions}

For the adhesively bonded lap jointed composites described.

- Joints with $30 \mathrm{~mm}$ bond length exhibit higher static and fatigue strength than those with 60 $\mathrm{mm}$. Fatigue strength is also improved using the stiffer laminate adherent $0 / 0$. 
- Immersion in water at $20^{\circ} \mathrm{C}$ causes a loss in static strength of about $30 \%$ after a period of $15-45$ days exposure. For water immersion at $40{ }^{\circ} \mathrm{C}$ a similar loss of static strength was observed after less than 15 days of water exposure.

- The effect of water exposure on the fatigue behaviour is mainly conditioned by the water temperature and to a lesser degree by the exposure time. The exposure in water at $70{ }^{\circ} \mathrm{C}$ the adhesive degrades significantly being above its service application limit.

- The first two stages of the reduction in dynamic stiffness (similar to that which occurrs in the base adherent composite) can be attributed to creep deformation. The joints are highly susceptible to creep for the stress ranges used in the fatigue tests including those carried out at room temperature. Creep results are reasonably "fitted" by the Manson-Haferd parameter.

\section{Acknowledgements}

The authors would thank RTA-NATO, Support Programme Project P-128, for funding most of the work reported.

\section{References}

[1] Renton WJ, Vinson JR. Fatigue behavior of bonded joints in composite material structures. J Aircraft 1975;12(5:):442-7.

[2] Kreiger RB. Fatigue testing of structural adhesives. SAMPE Proceedings 1979;24(1:):1102-25.

[3] Renton WJ, Vinson JR. The efficient design of adhesive bonded joints. J Adhesion 1975;7:175-93.

[4] Harris JA, Fay PA. Fatigue life evaluation of structural adhesives for automotive applications. Int J Adhes Adhes 1992;12(1:):9-18.

[5] Adams RD, Coppendale, J, Mallick, V, Al-Hamdan H. The effect of temperature on the strength of adhesive joints. Int $\mathbf{J}$ Adhes Adhes 1992;12(3):185-90.
[6] Cotter JL. Developments in adhesives - 1. [W. C. Wake, editor]. London: Applied Science Publishers Ltd 1977 [Chapter 1].

[7] Brewis DM, Comyn, J, Cope BC, Moloney AC. Effect of carriers on the performance of aluminium alloy joints bonded with an epoxide polyamide adhesive. Polymer 1990;21:344.

[8] Brewis DM, Comyn J, Tegg JL. The durability of some epoxide adhesive bonded joints on expositure to moist warm air. Int $\mathbf{J}$ Adhes Adhes 1980;1:35.

[9] Cognard J. Influence of water on the cleavage of adhesive joints. Int J Adhes Adhes 1988;8(2:):93-9.

[10] Davies P, Pomies F, Carlsson LA. Influence of water absorption on transverse tensile properties and shear fracture toughness of glass/polypropylene. J Compos Mat 1996;30(9:):1004-19.

[11] Chiou P, Bradley L. Effects of seawater absorption on the fatigue crack development in carbon/epoxy E.D.T. specimens. Composites 1995;26(12:):869-76.

[12] Guess TR, Alfred RE, Gestle Jr FP. Comparison of lap shear test specimens. J Testing Evaluation 1997;5(3):84-93.

[13] Mall S, Ramamurthy G. Effect of bond thickness on fracture and fatigue strength of adhesive bonded composite joints. Int $\mathbf{J}$ Adhes Adhes 1989;9(1:):33-7.

[14] Matthews FL, Tester TT. The influence of stacking sequence on the strength of bonded CFRP single lap joints. Int J Adhes Adhes 1985;5(1):13-18.

[15] Davis A, Sims D. Weathering of plastics. Applied Science Publishers; 1983. p. 276-85.

[16] Comyn J. The effect of water on adhesives and adhesive joints. Plastics and Rubber Processing and Applications 1983; 3:201-5.

[17] Bowditch MR. The durability of adhesive joints in the presence of water. Int J Adhes Adhes 1996;16(2):73-9.

[18] Ning Su, Mackie RI, Harvey WJ. The effects of ageing and environment on the fatigue life of adhesive joints. Int $\mathbf{J}$ Adhes Adhes 1992;12(2:):85-93.

[19] Ferreira JAM, Costa JDM, Reis PNB. Static and fatigue behaviour of glass-fibre-reinforced polypropylene composites. Theor Applied Fracture Mech 1999;31:67-74.

[20] Ferreira JAM, Costa JDM, Reis PNB, Richardson MOW. Analysis of fatigue and damage in glass-fibre-reinforced polypropylene composite materials. Composites Science and Technology 1999;59:1461-7.

[21] Dowling NE, Mechanical behavior of materials: engineering methods for deformation, fracture and fatigue. New Jersey: Prentice-Hall Int. Ed.; 1993. 